\title{
Daily or weekly dosing with EGFR inhibitors, gefitinib and lapatinib, and AKT inhibitor MK2206 in mammary cancer models
}

\author{
RONALD A. LUBET ${ }^{1}$, VERNON E. STEELE ${ }^{1}$, M.M. JULIANA ${ }^{2}$, ANN BODE ${ }^{3}$, \\ FARIBA MOEINPOUR $^{2}$ and CLINTON J. GRUBBS ${ }^{2}$
}

\author{
${ }^{1}$ Division of Cancer Prevention, National Cancer Institute, Bethesda, MD 20852; ${ }^{2}$ Department of Surgery, \\ University of Alabama at Birmingham, Birmingham, AL 35294; ${ }^{3}$ Division of Cancer Biomarkers and Drug Resistance, \\ University of Minnesota Hormel Institute, Austin, MN 55912, USA
}

Received July 24, 2017; Accepted January 12, 2018

DOI: $10.3892 / o r .2018 .6313$

\begin{abstract}
Daily vs. weekly dosing with EGFR inhibitors (gefitinib and lapatinib) and an AKT inhibitor (MK2206) were compared in two rodent breast cancer models. Female Sprague-Dawley rats were administered methylnitrosourea (MNU) at 50 days of age, and gefitinib (daily/weekly dosing at $10 / 70 \mathrm{mg} / \mathrm{kg} \mathrm{BW}$ ) or lapatinib (daily/weekly dosing at $75 / 525 \mathrm{mg} / \mathrm{kg} \mathrm{BW}$ ) were administered by gavage beginning 5 days after MNU. For the prevention studies, weekly or daily dosing with gefitinib or lapatinib reduced cancer multiplicity $>75 \%$, and all treatments reduced tumor weights by $>90 \%$. For the therapeutic studies, MNU-treated rats were followed until small palpable mammary cancers developed. The rats were then treated daily or weekly as above for 6 weeks. Either daily or weekly dosing with lapatinib or gefitinib caused regression in $>50 \%$ of the tumors. Immunohistochemistry biomarker studies in palpable mammary cancers following a weekly dose of gefitinib showed that 1 day (but not 7 days) after treatment, the levels of phosphorylated EGFR1 were significantly decreased. In an ER-negative (ER') Neu-overexpressing model employing MMTV-Neu/P53KO mice, daily (100 mg/kg BW/day, 5 days each week), or weekly dosing (500 or $250 \mathrm{mg} / \mathrm{kg} \mathrm{BW}$ ) with gefitinib reduced tumor multiplicity 65,85 and $75 \%$, respectively. In the MNU prevention model, daily dosing $(100 \mathrm{mg} / \mathrm{kg}$ BW/day) with the allosteric AKT inhibitor MK2206 was ineffective, while weekly dosing $(700 \mathrm{mg} / \mathrm{kg} \mathrm{BW})$ reduced the final tumor weight $>70 \%$. Combining weekly MK2206 with the aromatase inhibitor vorozole $(0.12 \mathrm{mg} / \mathrm{kg} \mathrm{BW} /$ day $)$ showed that each compound alone reduced tumor multiplicity $40-50 \%$. The combination reduced cancer multiplicity $\sim 70 \%$. These studies demonstrate the efficacy of weekly dosing with various
\end{abstract}

Correspondence to: Dr Clinton J. Grubbs, Department of Surgery, University of Alabama at Birmingham, Birmingham, AL 35294, USA

E-mail: clintongrubbs@uabmc.edu

Key words: cancer, prevention, therapy protein kinase inhibitors; raising the possibility of employing these agents in a breast cancer preventive setting.

\section{Introduction}

The EGFR pathway was defined more than 20 years ago, and was quickly shown to be associated with a variety of important cellular pathways (1); including those associated with cell proliferation and the cell cycle (2). The potential role of EGFR2 antagonists (e.g., trastuzumab and lapatinib) in the treatment of Neu-amplified breast cancer was immediately appreciated, and the use of the monoclonal antibody trastuzumab and more recently pertuzumab directed against Neu (EGFR2) has profoundly altered the outcome for women with Neu-amplified breast cancer (3). The potential use of an EGFR1 inhibitor appeared particularly appealing since EGFR1 is overexpressed in multiple tumor types (2). EGFR1 smallmolecule inhibitors are approved for the treatment of lung cancer (4), particularly lung tumors with EGFR1 mutations, and for pancreatic cancers (in conjunction with standard therapy in an advanced setting). In addition, small-molecule inhibitors or monoclonal antibodies directed against EGFR1 have been employed in head and neck cancer in conjunction with radiation, in colorectal cancer, and in second line therapy of squamous cell carcinoma of the lung $(5,6)$. In the treatment of estrogen receptor-positive $\left(\mathrm{ER}^{+}\right)$breast cancer, there have been two studies which demonstrate potential efficacy based either on clinical outcome in a neoadjuvant setting (7), or striking modulation of the generally accepted biomarker Ki67 in a pre-surgical protocol (8). Furthermore, recent data have demonstrated efficacy in advanced $\mathrm{ER}^{+}$breast cancer when used in conjunction with an aromatase inhibitor (9). We previously reported the EGFR inhibitors gefitinib (10) and erlotinib (11), as well as the Neu/EGFR2 inhibitor lapatinib (12), were effective in an MNU-induced model of $\mathrm{ER}^{+}$ mammary cancer when these agents were administered by daily dosing.

AKT, a serine threonine kinase, is the major downstream substrate for PI3K and helps regulate cellular processes that contribute to tumor progression; including cell proliferation, survival, tissue invasion and angiogenesis. MK2206, a highly selective allosteric inhibitor of AKT showed positive activity 
in a phase I study, and is currently undergoing clinical investigation in breast cancer patients (13).

Various animal models of breast cancer have routinely been employed in the testing for preventive agents. The $\mathrm{ER}^{+}$ rat mammary cancer model has been used for more than 30 years (14). These cancers are histologically and genetically similar to well-differentiated $\mathrm{ER}^{+}$human breast cancers (15). Furthermore, as may be expected, treatments that alter the hormonal axis (e.g., SERMS, aromatase inhibitors) were found to be strong chemopreventive and even therapeutic agents in this model $(16,17)$. MNU-induced mammary cancers, as mentioned above, respond to EGFR inhibitors $(10,11)$. The second model that was employed was the mouse MMTV-Neu/P53KO that was initially developed by Muller et al (18). These animals develop ER ${ }^{-}$Neu-overexpressing mammary carcinomas. This model was shown to be particularly sensitive to the effects of EGFR inhibitors (19). We used a slight modification of the model which includes a heterozygous KO of P53 (20). Cancers from this model parallel human $\mathrm{ER}^{-} \mathrm{Neu}$-amplified tumors which tend to have P53 mutations.

Although (as will be shown below) weekly dosing with TKI inhibitors is at least equally effective with daily dosing, perhaps the main advantage relates to the observation that weekly dosing with EGFR inhibitors has clearly been shown to be less toxic. Although the targeted kinase inhibitors are clinically important, questions of dosing schedule and toxicity (21) are likely to inhibit their use; particularly in a prevention setting. In addition, there are specific feedback loops (see Discussion) associated with daily treatment with these classes of agents that may be partially averted with weekly dosing. We recently showed in the MNU model that erlotinib was effective when administered daily or weekly (10). In the present study, we expanded this observation to additional EGFR inhibitors (gefitinib and lapatinib) to determine whether weekly dosing is effective in a second mammary cancer model (MMTV-Neu/P53KO) which is ER-. Specifically, we determined: i) the preventive efficacy of daily and weekly dosing with gefitinib and lapatinib in the MNU-induced $\mathrm{ER}^{+}$model; ii) the efficacy of daily and weekly therapeutic dosing in the MNU-induced $\mathrm{ER}^{+}$model; iii) the efficacy of daily and weekly gefitinib in the MMTV-Neu/P53KO model; and iv) the modulation of biomarkers by weekly dosing with gefitinib in the MNU model when examined 1 day or 7 days after a weekly dose. These studies demonstrated that weekly dosing was effective in both models, and that proliferation-related biomarkers were modulated either 1 day or even 7 days following a weekly dose with gefitinib. Finally, we determined the more general relevance of the altered dosing to other small-molecule kinase inhibitors. Specifically, the allosteric inhibitor AKT MK2206 given alone or together with the aromatase inhibitor (vorozole) was tested following daily or weekly dosing.

\section{Materials and methods}

Chemicals and animals. Treatment of female Sprague-Dawley rats was conducted as previously described $(10,11)$. Methylnitrosourea (MNU) was obtained from the NCI Chemical Carcinogen Repository, and injected i.v. (75 mg/kg BW) via the jugular vein when the animals were 50 days of age. Teklad diet and rats were obtained from Harlan Sprague-Dawley,
Inc. (Indianapolis, IN, USA). All rats and mice were housed in IACUC approved (no. 09804) animal facilities at the University of Alabama at Birmingham. The use of animals was necessary to obtain data that would support a clinical trial of these agents in women. Ethical approval was given bt the IACUC Committee. Gefitinib, lapatinib and MK2206 were supplied by the NCI Cancer Prevention Repository. All agents were administered by gavage on a daily or weekly basis. Agents were administered in a volume of $0.5 \mathrm{ml} /$ gavage for rat studies, and $0.2 \mathrm{ml} /$ gavage for mouse studies. The vehicle for both agents was ethanol:polyethylene glycol 400 $(10: 90 ; \mathrm{v} / \mathrm{v})$.

Data collection and analyses. In all studies, rats and mice were palpated for mammary tumors twice weekly and weighed 1 time/week. Body weights of the rats or mice did not differ $>5 \%$ from the controls in either the prevention or therapeutic studies. Statistical analyses of cancer incidence and latency were determined using log-rank analysis, while differences in final cancer multiplicity and in final tumor weights were determined by Wilcoxon rank test $(10,11)$.

Prevention studies with EGFR inhibitors. In the prevention studies, treatment of the rats with gefitinib or lapatinib was initiated 5 days after MNU administration (or at 55 days of age). The number of rats/group was 15 . We had previously determined effective daily doses for gefitinib (11) and lapatinib (12). Gefitinib was administered daily at a dose of $10 \mathrm{mg} / \mathrm{kg} \mathrm{BW} /$ day, while the weekly dose was 7 times the daily dose (70 mg/kg BW). Lapatinib was administered daily at a dose of $75 \mathrm{mg} / \mathrm{kg}$ BW/day, while the weekly dose was 7 times the daily dose $(525 \mathrm{mg} / \mathrm{kg} \mathrm{BW})$. These doses were all less than the daily human equivalent dose (HED) based on FDA scaling; which would be roughly 12 and $120 \mathrm{mg} / \mathrm{kg} \mathrm{BW} /$ day for gefitinib and lapatinib, respectively. Rats were examined twice a week for the development of palpable mammary tumors. At the end of the study, tumors were weighed and submitted for histological evaluation.

Therapeutic study. Rats received MNU at 50 days of age and were observed for the appearance of mammary cancers. When an animal developed a tumor of $\sim 100-200 \mathrm{~mm}^{2}$, the rat received lapatinib (75 mg/kg BW/day or $525 \mathrm{mg} / \mathrm{kg} \mathrm{BW}$, once/week) or gefitinib (10 mg/kg BW/day or $70 \mathrm{mg} / \mathrm{kg} \mathrm{BW}$, once/week) for 6 weeks. Tumor size was determined with calipers before initiation of treatment and twice each week during the course of treatment. The largest diameter of the cancer was measured and this value was multiplied by the perpendicular diameter (size expressed in $\mathrm{mm}^{2}$ ). The gefitinib and lapatinib treated rats as well as the control group had an $n$ of $10(10,11,22)$.

MMTV-Neu/P53KO (ER') mouse mammary cancer model. $\mathrm{MMTV}-\mathrm{Neu}^{+/-} / \mathrm{p} 53 \mathrm{KO}^{+-}$mice were generated and maintained as previously described (23). MMTV-Neu transgenic mice [strain FVB/N-Tg (MMTV-Neu) $202 \mathrm{Mul} / \mathrm{J}$ ] were purchased from the Jackson Laboratory (Bar Harbor, ME, USA). A p53 deficient line (p53 NS-T) was purchased on a C57BL background (Taconic) and back-crossed at least 5 times onto a FBV/N background. For generation of MMTV-Neu ${ }^{+/ /} / \mathrm{p} 53$ $\mathrm{KO}^{+/-}$females, $\mathrm{p}^{-/-}$males were crossed with MMTV-Neu ${ }^{+/+}$ 


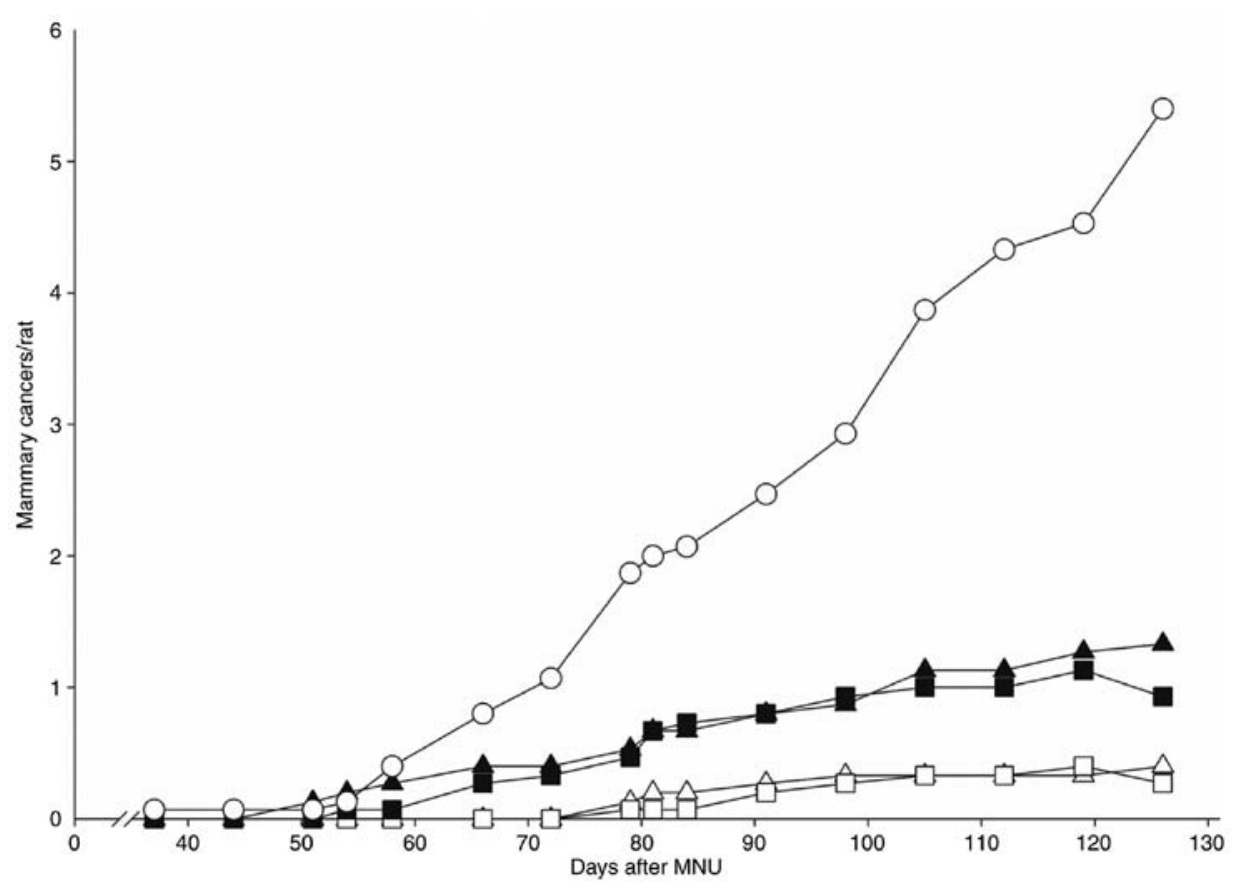

Figure 1. Effects of daily or weekly dosing with lapatinib or gefitinib on the prevention of $\mathrm{ER}^{+} \mathrm{MNU}$-induced cancers. Rats (n=15/group) were treated with $\mathrm{MNU}$ at 50 days of age, and 5 days later, the rats were treated with lapatinib $(75 \mathrm{mg} / \mathrm{kg}$ BW/day or $525 \mathrm{mg} / \mathrm{kg} \mathrm{BW}, 1$ time/week) or gefitinib (10 mg/kg BW/day or $70 \mathrm{mg} / \mathrm{kg}$ BW, 1 time/week). Following either dosing regimen, the agent's tumor multiplicity was significantly reduced $(\log$-rank, $\mathrm{P}<0.025)$ at termination of the study. $\square$ Lapatinib daily; lapatinib weekly; $\Delta$ gefitinib daily; $\boldsymbol{\Lambda}$ gefitinib weekly.

females. MMTV-Neu females were generated by crossing MMTV-Neu ${ }^{+++}$males with FVB/NJ females. The animal rooms were lighted $12 \mathrm{~h} /$ day in a facility specially designed for administering chemical carcinogens to animals. Mice were given diet (Teklad 4\% mash) and water ad libitum. Mice were treated with gefitinib by gavage either daily (5 times/week) or weekly beginning at 6 weeks of age. Mice were examined weekly for the development of palpable mammary tumors beginning at 4 months of age. Mice were sacrificed when a large palpable mammary tumor developed or at termination when mice were 11 months of age.

Prevention studies of MK2206 alone or with vorozole in the $M N U$-induced rat model. In the first study, rats were treated with MK2206 administered daily $(33,100$ or $300 \mathrm{mg} / \mathrm{kg}$ BW/day) or weekly ( $700 \mathrm{mg} / \mathrm{kg} \mathrm{BW})$ initiated 5 days after MNU administration (or at 55 days of age). Based on these initial studies showing the efficacy of weekly dosing with MK2206, weekly dosing of this agent together with a low dose of the aromatase inhibitor vorozole $(0.12 \mathrm{mg} / \mathrm{kg} \mathrm{BW} /$ day $)$ was evaluated. We had previously shown that vorozole at this dose was highly effective in this model (17).

Biomarker levels in treated MNU-induced tumors. Cancers were allowed to develop in MNU-treated rats. When a palpable tumor developed in an animal, it was treated with gefitinib (70 mg/kg BW). All tumor-bearing rats were administered gefitinib or vehicle on day 0 . Seven days later, one set of rats was sacrificed. The second group of rats was administered a second dose of gefitinib on day 6 and sacrificed $24 \mathrm{~h}$ later. At the time of sacrifice, mammary cancers were excised and fixed for histological classification and immunohistochemistry (IHC).
IHC detection of phosphorylated EGFR or Ki67. Paraffinembedded tissue samples were heated, and paraffin was removed with xylene. Rehydration of the samples was performed using ethanol, and antigen retrieval was performed using $10 \mathrm{mM}$ sodium citrate buffer ( $\mathrm{pH}$ 6.0). Endogenous peroxidase was quenched using $3 \% \mathrm{H}_{2} \mathrm{O}_{2}$ /methanol. Specimens were blocked using $50 \%$ goat serum/1X PBS for $1 \mathrm{~h}$. Ki-67 (ab-16667; Abcam, Cambridge, UK) was diluted 1:100 in 50\% goat serum/1X PBS. The antibody to detect p-EGFR (Tyr.1173; 4407; Cell Signaling Technology, Beverly, MA, USA) was diluted 1:100 in 50\% goat serum/1X PBS. The primary antibody was incubated overnight at $4^{\circ} \mathrm{C}$, and washed with PBS for $5 \mathrm{~min}$. The biotinylated anti-rabbit secondary antibody (Ba-1000; Vector Laboratories, Inc., Burlingame, CA, USA) was prepared at a 1:150 dilution. Samples were incubated with the secondary antibody for $60 \mathrm{~min}$. The ABC complex/HRP solution (Pk-6100; Vector Laboratories) was applied and incubated for $30 \mathrm{~min}$ at room temperature. Slides were then washed with $1 \mathrm{X}$ PBS for 3 min twice. Slides were incubated until staining was sufficient and then rinsed in deionized water. Counterstaining was performed by placing the slides in hematoxylin for $1 \mathrm{~min}$. Dehydration was performed by dipping slides in ethanol followed by xylene for $7 \mathrm{~min}$ each. The images of the slides were acquired using a Leica Microscope DMIRB (type 090-132.701) and Image Pro 6.3, and then analyzed using Image Pro Premier 9.0 software (Media Cybernetics, Inc., Bethesda, MD, USA).

\section{Results}

Preventive efficacy of weekly dosing with gefitinib or lapatinib in the MNU model. Our previous studies showed that gefitinib at a dose of $10 \mathrm{mg} / \mathrm{kg}$ BW/day and lapatinib at a dose 
Table I. Effect of daily or weekly treatment with gefitinib or lapatinib on development of MNU-induced mammary tumors in rats.

\begin{tabular}{lccc}
\hline & \multicolumn{3}{c}{ Mammary cancers } \\
\cline { 2 - 4 } Treatment (no. of rats/group=15) & Incidence (\%) & Multiplicity & Tumor weight (g) \\
\hline Vehicle & 94 & 4.9 & 8.49 \\
Gefitinib (daily, $10 \mathrm{mg} / \mathrm{kg} \mathrm{BW} /$ day) & $27^{\mathrm{b}}$ & $0.27^{\mathrm{b}}$ & $0.81^{\mathrm{b}}$ \\
Gefitinib (weekly, 70 mg/kg BW) & $56^{\mathrm{a}}$ & $1.0^{\mathrm{b}}$ & $1.07^{\mathrm{b}}$ \\
Lapatinib (daily, 75 mg/kg BW/day) & $40^{\mathrm{b}}$ & $0.4^{\mathrm{b}}$ & $0.26^{\mathrm{b}}$ \\
Lapatinib (weekly, 525 mg/kg BW) & 66 & $1.3^{\mathrm{b}}$ & $1.2^{\mathrm{b}}$ \\
\hline
\end{tabular}

Treatment was initiated 5 days following MNU administration. ${ }^{\mathrm{a}} \mathrm{P}<0.05 ;{ }^{\mathrm{b}} \mathrm{P}<0.025$; based on Wilcoxon rank of the treatment group compared to the vehicle control at the end of the study.

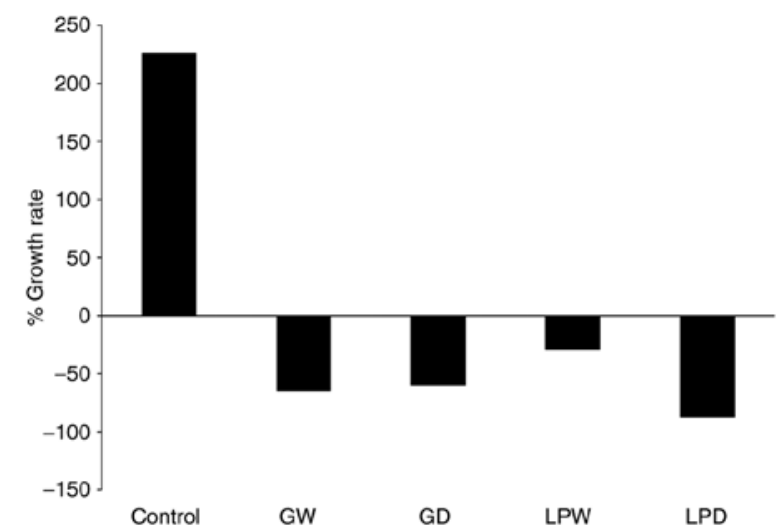

Figure 2. Effects of daily or weekly dosing with lapatinib or gefitinib on therapy of $\mathrm{ER}^{+} \mathrm{MNU}$-induced mammary cancers. Rats were treated with MNU at 50 days of age, and small palpable mammary lesions were allowed to develop. At that time, cancer-bearing rats ( $n=10 /$ group) were treated with lapatinib (75 mg/kg BW/day or $525 \mathrm{mg} / \mathrm{kg} \mathrm{BW,} 1$ time/week) or gefitinib (10 mg/kg BW/day or $70 \mathrm{mg} / \mathrm{kg} \mathrm{BW}, 1$ time/week). The bars show average growth for the various treatment groups. Overall growth in control tumors vs those in each of the treatment groups was decreased (Wilcoxon rank analysis, $\mathrm{P}<0.025)$. GW, gefitinib weekly; GD, gefitinib daily; LM, lapatinib weekly; LPD, lapatinib daily.

of $75 \mathrm{mg} / \mathrm{kg}$ BW/day were each highly effective in preventing $\mathrm{ER}^{+}$mammary cancers $(11,12)$. We, therefore, compared the efficacy of daily dosing with weekly dosing at 7 times the daily dose. Daily lapatinib (75 mg/kg BW/day) was highly effective, reducing tumor multiplicity by $85 \%$. A weekly dose of $525 \mathrm{mg} / \mathrm{kg} \mathrm{BW}$ reduced tumor multiplicity $\sim 75 \%$. Results with both doses were significantly different from the MNU controls $(\mathrm{P}<0.02)$, and the outcomes of the two treatments did not differ from each other. Similarly, gefitinib was tested either daily (10 mg/kg BW/day) or weekly (70 mg/kg BW). The daily dose inhibited tumor multiplicity by $80 \%$, whereas the weekly dose was $70 \%$ effective (Fig. 1). Measurements of final tumor weights (Table I) showed that either daily or weekly dosing with gefitinib or lapatinib reduced the average tumor weight by $90 \%$ when compared to the tumors from the control rats.

Therapeutic efficacy of weekly dosing with gefitinib or lapatinib in the MNU model. The MNU mammary cancer model was also used to examine the therapeutic efficacy of lapatinib or gefitinib by allowing small palpable tumors to develop before initiating treatment (Fig. 2). Tumor-bearing rats (10/group) were treated with lapatinib $(75 \mathrm{mg} / \mathrm{kg}$ BW/day) or gefitinib $(10 \mathrm{mg} / \mathrm{kg}$ $\mathrm{BW} /$ day) on a daily basis, or with lapatinib $(525 \mathrm{mg} / \mathrm{kg} \mathrm{BW})$ or gefitinib (70 mg/kg BW) on a weekly basis, or received the vehicle. Cancers in the vehicle-treated group increased in size by $250 \%$ over 6 weeks. Daily or weekly treatments with gefitinib or lapatinib reduced tumor size on average by $35-50 \%$. Daily or weekly dosing of either inhibitor resulted in at least half of the treated mammary tumors decreasing by $60 \%$ in size. We considered a $60 \%$ decrease as a clear regression; implying that either daily or weekly dosing was highly effective.

Preventive efficacy of weekly dosing with gefitinib in the MMTV/Neu transgenic model. The efficacy of gefitinib in the MMTV-Neu model was evaluated. Gefitinib was either administered at $100 \mathrm{mg} / \mathrm{kg} \mathrm{BW/day,} 5$ times/week or administered weekly at 500 or $250 \mathrm{mg} / \mathrm{kg} \mathrm{BW}$ beginning when the mice were 35 days of age. The studies examining daily and weekly treatments were performed at different times. Daily dosing with gefitinib yielded a $65 \%$ decrease in tumor multiplicity, and the weekly dosing at 500 or $250 \mathrm{mg} / \mathrm{kg} \mathrm{BW}$ both reduced tumor multiplicity $75-80 \%$ (Fig. 3). There is probably no significant difference from the daily dosing; however, since the studies were performed at different times a direct comparison could not be made.

Preventive efficacy of weekly dosing with the AKT-inhibitor MK2206 in the MNU rat model. In the initial studies, the preventive effects of MK2206 alone were determined in the MNU model. Daily doses of 30, 100 and $300 \mathrm{mg} / \mathrm{kg} \mathrm{BW/day}$ or a weekly dose of $700 \mathrm{mg} / \mathrm{kg}$ BW were administered. The daily dose of $300 \mathrm{mg} / \mathrm{kg} \mathrm{BW/day} \mathrm{was} \mathrm{toxic,} \mathrm{while} \mathrm{the} \mathrm{two}$ lower daily doses were ineffective in preventing mammary cancers. In contrast, the single weekly dose of $700 \mathrm{mg} / \mathrm{kg}$ BW was moderately effective; substantially increasing tumor latency [log-rank $\mathrm{P}<0.05$ (Fig. 4A)] and decreasing final tumor weights (Table IIA). This weekly dosing did not cause any observable toxicity. In the second study, we examined the effects of weekly dosing of MK2206, daily dosing with a low dose of the aromatase inhibitor vorozole, or the combination of these agents (Fig. 4B). Weekly MK2206 or vorozole reduced tumor multiplicity $40-50 \%$, and the combination was effective 

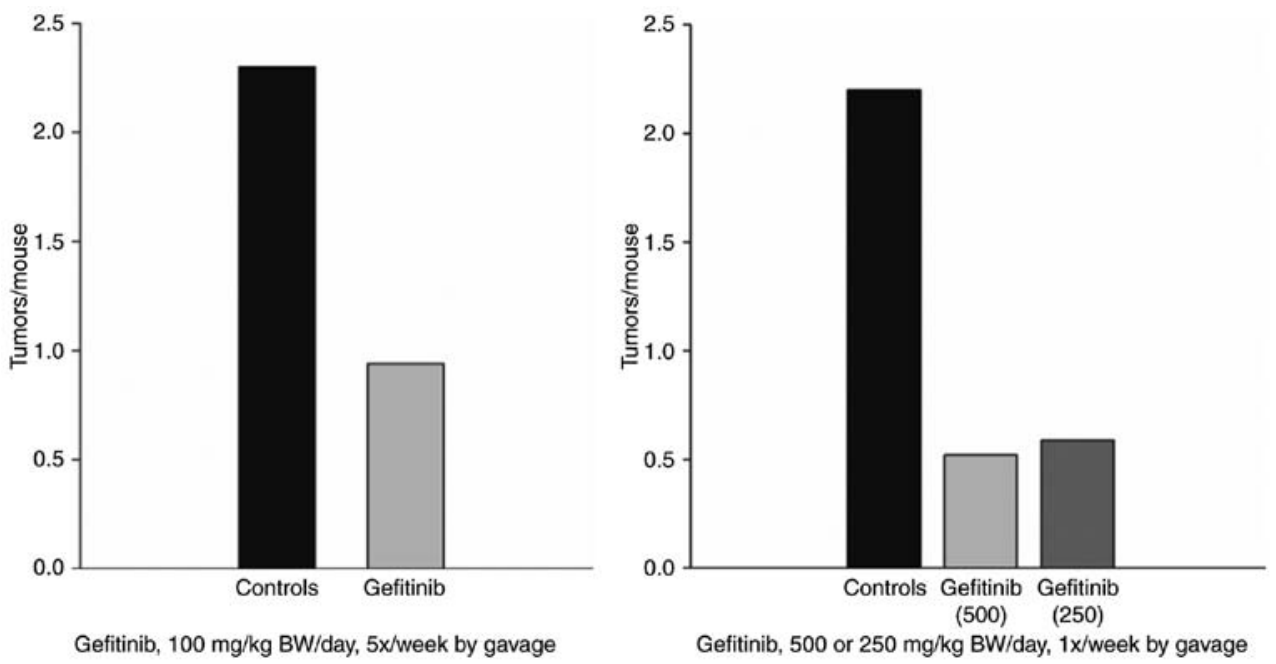

Figure 3. Effects of daily or weekly dosing with gefitinib on prevention of ER- mammary cancers in transgenic MMTV-Neu/P53KO mice. Mice (25/group) were treated with gefitinib (100 mg/kg BW/day, 5 days/week or 250 or $500 \mathrm{mg} / \mathrm{kg} \mathrm{BW,} 1$ time/week). Animals were followed until they developed a large palpable tumor or until 7 months of age. Daily dosing with gefitinib or weekly dosing at either 250 or $500 \mathrm{mg}$ reduced multiplicity (log-rank, $\mathrm{P}<0.05$ ).
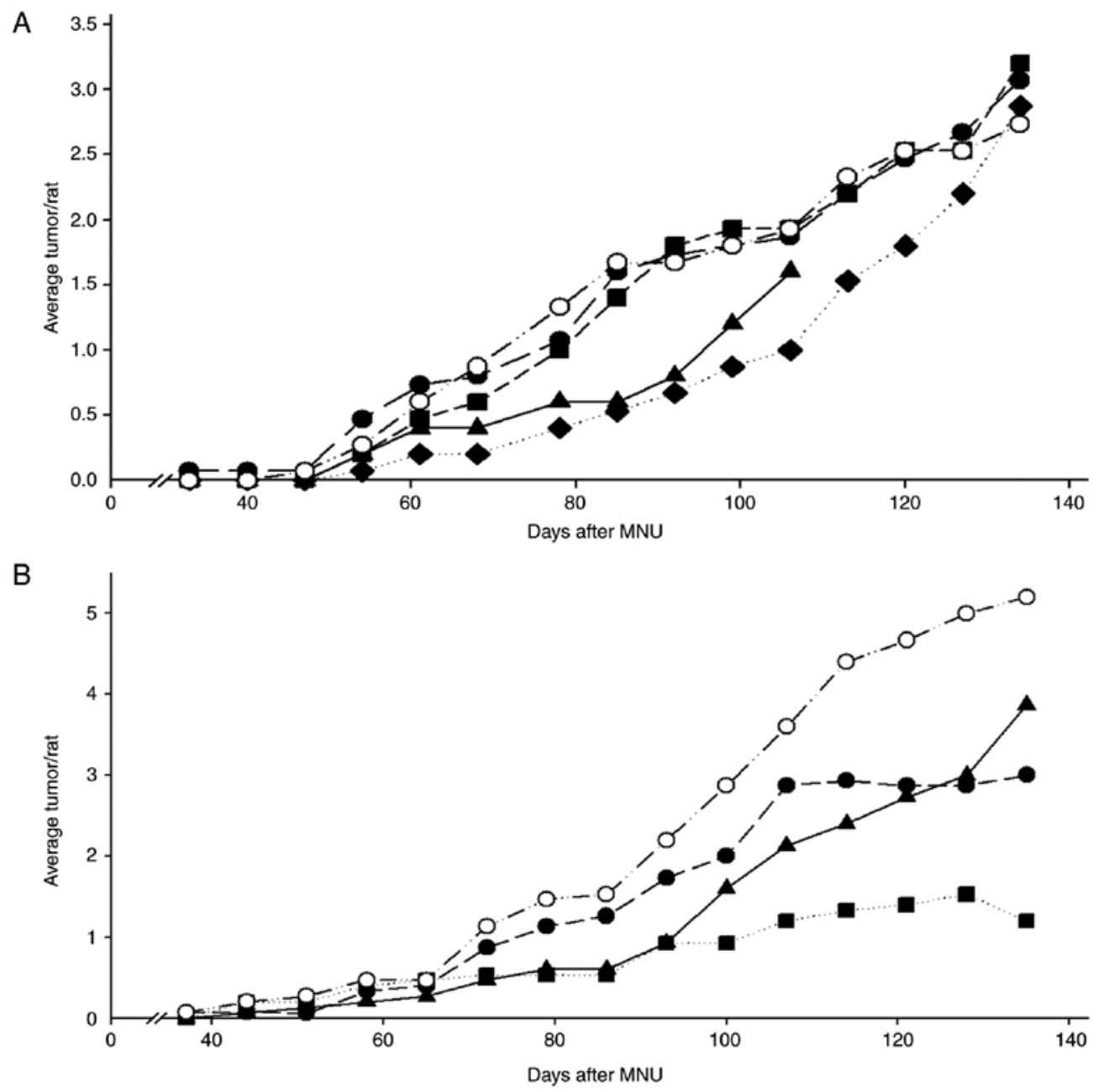

Figure 4. (A and B) Effects of daily or weekly dosing with MK2206 and the effects of weekly dosing with MK2206 and vorozole on the development of $\mathrm{ER}^{+}$tumors. Rats ( $\mathrm{n}=15 / \mathrm{group}$ ) were treated with $\mathrm{MNU}$ at 50 days of age and, beginning 5 days later, treated with the indicated agents. (A) Rats were treated with MK2206 (30, 100 or $300 \mathrm{mg} / \mathrm{kg}$ BW/day or $700 \mathrm{mg} / \mathrm{kg} \mathrm{BW}, 1$ time $/$ week). Daily doses of $33 \mathrm{or} 100 \mathrm{mg} / \mathrm{kg} \mathrm{BW} / \mathrm{day}$ were ineffective, while the $300 \mathrm{mg} / \mathrm{kg}$ BW/day was toxic. The dose of $700 \mathrm{mg} / \mathrm{kg} \mathrm{BW}, 1$ time/week reduced the development of cancers (log-rank, $\mathrm{P}<0.05) . \circ \mathrm{MK} 2206$ (30 mg/kg BW/day); • MK2206 $(100 \mathrm{mg} / \mathrm{kg} \mathrm{BW} /$ day); $\Delta$ MK2206 (300 mg/kg BW/day); MK2206 (700 mg/kg BW/week); and $\boldsymbol{\bullet}$ controls. Tumor weights in this group were decreased $\sim 70 \%$; Wilcoxon rank, P<0.05 (Table IIA). (B) Rats were treated with MK2206 (700 mg/kg BW, 1 time/week) and/or a suboptimal dose of the aromatase inhibitor vorozole $(0.12 \mathrm{mg} / \mathrm{kg} \mathrm{BW} / \mathrm{day})$. Each agent alone reduced tumor multiplicity $30-40 \%$, while the combination decreased the development of tumors $\sim 70 \%$ (Wilcoxon rank, $\mathrm{P}<0.05)$. ^ MK2206 (700 mg/kg BW/week); • vorozole $(0.12 \mathrm{mg} / \mathrm{kg}$ BW/day); - MK2206+vorozole; o controls. Tumor weights in this group were also decreased; Wilcoxon rank, ${ }^{*} \mathrm{P}<0.05$ (Table IIB). 
Table II. Effect of AKT inhibitor MK2206 and/or vorozole on the development of MNU-induced mammary cancers in rats.

A, Effect of daily or weekly treatment with the AKT inhibitor MK2206

Mammary cancers

\begin{tabular}{lccc} 
Treatment (no. of rats/group=15) & Incidence $(\%)$ & Multiplicity & Weight $(\mathrm{g})$ \\
\hline Vehicle & 94 & 2.7 & 6.6 \\
MK2206 (daily, $33 \mathrm{mg} / \mathrm{kg} /$ day) & 87 & 3.0 & 6.6 \\
MK2206 (daily, $100 \mathrm{mg} / \mathrm{kg} / \mathrm{day})$ & 87 & 3.2 & 3.25 \\
MK2206 (weekly, $700 \mathrm{mg} / \mathrm{kg}$ ) & 80 & 2.8 & $1.57^{\mathrm{a}}$ \\
\hline
\end{tabular}

B, Effect of treatment with the AKT inhibitor MK2206 and/or daily treatment with vorozole

\begin{tabular}{lrrr}
\hline Vehicle & 100 & 4.5 & 9.1 \\
Vorozole (daily, $0.12 \mathrm{mg} / \mathrm{kg} /$ day) & 87 & 3.0 & 6.8 \\
MK2206 (weekly, $700 \mathrm{mg} / \mathrm{kg}$ ) & 87 & 3.2 & 5.9 \\
MK2206 (weekly, $700 \mathrm{mg} / \mathrm{kg}$ ) & 60 & $1.7^{\mathrm{a}}$ & $3.6^{\mathrm{a}}$ \\
+ vorozole (daily, $0.12 \mathrm{mg} / \mathrm{kg}$ day) & & & \\
\hline
\end{tabular}

Treatment was initiated 5 days following MNU administration. ${ }^{a} \mathrm{P}<0.05$, based on Wilcoxon rank of the treatment group compared to the vehicle control at the end of the study.
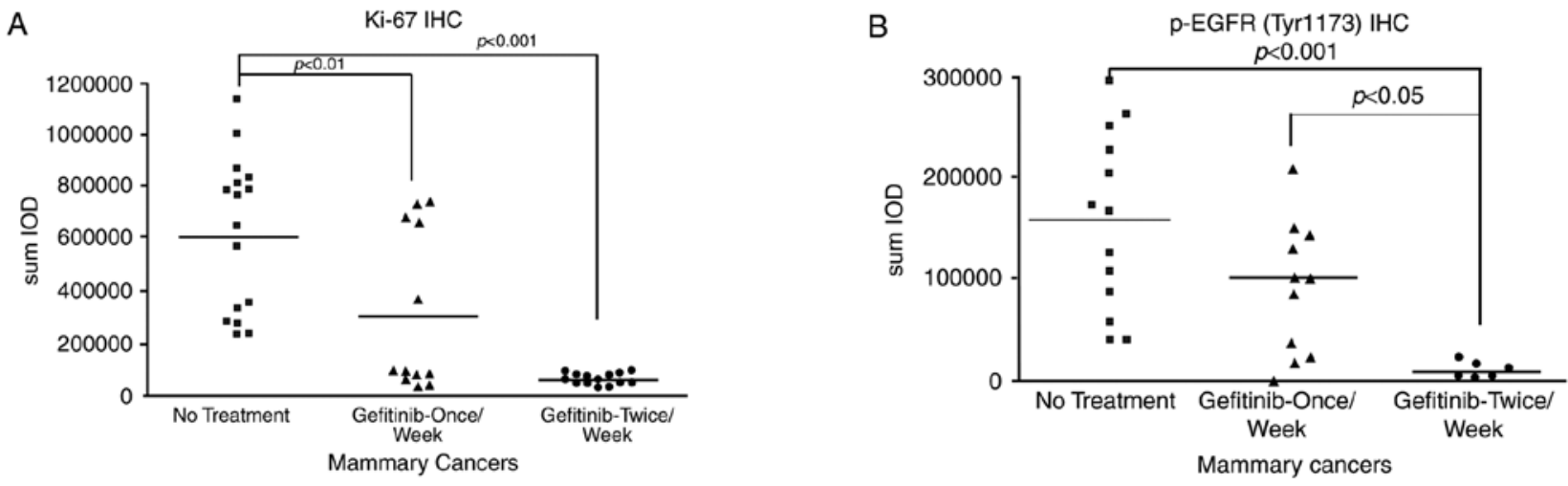

Figure 5. (A and B) Effects of weekly dosing with gefitinib on Ki67 levels or EGFR phosphorylation: results, 1 vs. 6 days post treatment (see Materials and methods). MNU-treated animals were allowed to develop small palpable tumors. At that time, rats were treated with a weekly dose of gefitinib (70 mg/kg BW) or vehicle control. Half of the gefitinib rats were sacrificed on day 6 (144 h post-single weekly gefitinib dosing). The other half of the gefitinib-treated tumor-bearing rats were administered a second weekly gefitinib dose and sacrificed $24 \mathrm{~h}$ later. IHC was determined employing specific antibodies directed against Ki67 or phosphorylated EGFR1. Methods and statistical analysis are described in Materials and methods.

in reducing multiplicity by $70 \%(\mathrm{P}<.05)$ and reducing tumor weights (Table IIB).

Modulation of biomarkers in tumor-bearing rats treated short term with gefitinib. Palpable tumors were treated with gefitinib (70 mg/kg BW, 1X/week) or vehicle on day 0. Mammary cancers were collected from animals administered one weekly dose of gefitinib and sacrificed on day 7, or from rats administered a second dose of gefitinib on day 6 and sacrificed $24 \mathrm{~h}$ later. We initially determined Ki67 levels in the cancers and found that in rats sacrificed 1 day or 7 days following a weekly dose of gefitinib significantly decreased levels were noted relative to the vehicle-treated rats. The results were more striking at $24 \mathrm{~h}$ after a weekly dose (Fig. 5A).
The effects of gefitinib treatment on endpoints more directly related to its mechanism of action were also examined; specifically, phosphorylation of EGFR. Levels of phosphorylated EGFR were profoundly reduced in mammary cancers obtained within 1 day of the last weekly dosing (Fig. 5B), but were not significantly reduced 6 days after a weekly dosing.

\section{Discussion}

The EGFR pathway is integral to various pathways of cell signaling and cell replication, and is overexpressed in various tumors including lung, head and neck, urinary bladder and triple-negative breast cance $(1,2)$. The primary focus of inhibiting this pathway has been the development of 
small-molecule inhibitors (gefitinib, erlotinib) or monoclonal antibodies (e.g., cetuximab, trastuzumab). EGFR1 inhibitors (gefitinib and erlotinib) have proven to be highly effective against a subset of lung adenocarcinomas that have mutations in the EGFR1 gene (4). In early ER ${ }^{+}$breast cancer, EGFR1 inhibitors have proven effective when administered together with aromatase inhibitors in $\mathrm{ER}^{+}$breast cancers (9). Notably, there are at least two studies implying the efficacy of EGFR inhibitors in earlier stages of $\mathrm{ER}^{+}$breast cancer. One was a true neoadjuvant study in which Iressa ${ }^{\circledR}$ administered for $12-16$ weeks (7) induced regression in $40 \%$ of tumors. The second study (8) showed significant decreases in proliferation in tumors treated short-term with Iressa. Inhibition of Ki67 has proven to be a highly predictive endpoint in the therapeutic setting of $\mathrm{ER}^{+}$breast cancer (24). The efficacy of EGFR inhibitors in $\mathrm{ER}^{+}$breast cancer is surprising since $\mathrm{ER}^{+}$tumors generally have low expression of EGFR1. EGFR2 (Neu) inhibitors have been shown to be a striking target for $\mathrm{ER}^{+}$or $\mathrm{ER}^{-}$breast cancers with amplification and overexpression of the Neu gene (3).

Previously we found that $\mathrm{MNU}$-induced $\mathrm{ER}^{+}$rat mammary cancers are highly sensitive to the EGFR1 inhibitors erlotinib and gefitinib (10,11), and the EGFR 2/1 inhibitor lapatinib (12). In our earlier prevention studies, these EGFR inhibitors caused a dosedependent decrease in tumor multiplicity and final tumor weights at dose levels below their human equivalents. Furthermore, these agents proved to be therapeutic in this model. All 3 agents exhibit toxicity in humans causing both acneiform skin rashes and diarrhea. However, these toxicities are not seen in either mice or rats at the effective doses. The primary impetus for examining the potential of weekly dosing with gefitinib or erlotinib is that weekly dosing with these agents at doses up to 7 times the daily dose has been shown to significantly decrease the incidence of toxicities, i.e., rash and diarrhea $(25,26)$. Thus, whether weekly dosing achieves significant efficacy it may allow for the use of these targeted agents in a prevention or adjuvant setting. One specific example is the recent finding that the combination of erlotinib plus sulindac was highly effective in individuals with familial adenomatous polyposis (27). A weekly dosing of the EGFR inhibitor which may be expected to reduce toxicity may make such a protocol more tolerable.

In the present experiments, we found in the MNU model that lapatinib and gefitinib are highly effective when administered weekly, as contrasted with daily dosing. This result may not be as surprising regarding gefitinib since the mechanistically similar EGFR1 inhibitor erlotinib was highly effective (10). The results with lapatinib which preferentially inhibits EGFR2 (Neu) and has a relatively short half-life in rodents $\left(\mathrm{T}_{1 / 2} \leq 4 \mathrm{~h}\right)$ is more surprising. Thus, lapatinib may be expected to achieve optimal serum levels at $\leq 48 \mathrm{~h}$; even with a weekly dose 7 times higher than the daily dose.

Weekly dosing of this class of agents was highly effective in the MMTV-Neu model of ER mammary cancer in transgenic mice. Notably, Brown et al previously demonstrated that the EGFR inhibitor gefitinib was profoundly effective in this model (19). The present data confirmed these results, and furthermore showed that weekly dosing at either 5 times or even 2.5 times the daily dose was as effective as daily dosing.

The effects of weekly dosing with gefitinib on expression of Ki67 in MNU-induced mammary cancers were examined. We previously showed that daily dosing with gefitinib decreased the levels of Ki67 in mammary tumors (11). When the effects of weekly dosing with gefitinib were determined, significant decreases in $\mathrm{Ki} 67$ at either 1 day or 7 days following a weekly dose were observed. Our specific interest in Ki67 labeling in mammary cancer is based on the fact that it appears to be indicative of agent efficacy in a preoperative setting in humans (24). When EGFR phosphorylation was examined 1 day after a weekly dose of gefitinib, a profound inhibition of EGFR1 phosphorylation was observed as expected. In contrast, most of this inhibitory activity was not observed 7 days following a single weekly dose. This would make sense if serum and cancer tissue levels of the agents were lower after this time period.

Given the efficacy of weekly dosing with the EGFR inhibitors, it was determined whether such an effect may be observed with other kinase inhibitors. The efficacy of MK2206, an inhibitor of the serine-threonine kinase AKT, was evaluated with or without the aromatase inhibitor vorozole in the MNU-induced model. Initially, the preventive agent showed that daily dosing of $100 \mathrm{mg} / \mathrm{kg} \mathrm{BW} /$ day or less was ineffective, while a weekly dose of $700 \mathrm{mg} / \mathrm{kg} \mathrm{BW}$ increased tumor latency and substantially decreased final tumor weights. In the second prevention study, the combination of MK2206 (700 mg/kg BW/week) was evaluated together with a low $(0.12 \mathrm{mg} / \mathrm{kg} \mathrm{BW} /$ day $)$ dose of the aromatase inhibitor vorozole. We performed numerous studies with vorozole in this model, and demonstrated it to have dose-dependent preventive (17) and therapeutic effects (22). The individual dosing with suboptimal vorozole and/or weekly MK2206 reduced tumor multiplicity $40-50 \%$, while the combination yielded a $70 \%$ decrease in tumor multiplicity and final tumor weights. This result has two important aspects. First, it confirms the general finding that this agent or class of agents has limited activity on its own, but appears more effective in combination with agents that inhibit estrogen activity. Additionally, it showed that weekly dosing was, in fact, more effective than daily dosing using the same total dose. Perhaps most importantly, this models clinical trials in tamoxifen-resistant $\mathrm{ER}^{+}$tumors combining an aromatase inhibitor and MK-2206 (13).

The other major question raised by the chemopreventive efficacy of weekly dosing is related to the responsible mechanism. One possibility is that the high dose level may hit additional targets. However, these agents are relatively target specific and, even if the higher dose hits additional targets for a short time, one is likely to achieve the higher serum levels only transiently. The second possibility may be that the pharmacokinetics of weekly dosing is markedly different. Certainly our prior study with erlotinib (10) showed a similar half-life for the drug; employing either daily or weekly doses ( 7 times the daily dose). Furthermore, our present data examining EGFR phosphorylation following gefitinib treatment imply that the primary pharmacologic effect (inhibition of EGFR phosphorylation) is lost over a weekly period. The third possibility is that there are feedback mechanisms that inhibit activity following continual exposure to an inhibitor. Thus, the increased efficacy of weekly dosing with the AKT inhibitor has a strong mechanistic rationale. Daily dosing with an AKT inhibitor produces a negative feedback loop that results in increased expression and phosphorylation of multiple proteins, including HER3, IGF1R and MEK (28). 
This negative feedback is likely to suppress the activity of AKT inhibitors. The weekly dosing with the AKT inhibitor (resulting in intermittent inhibition of AKT phosphorylation) is likely to partially ameliorate this feedback loop and, thereby, may actually increase efficacy. These negative feedback loops may not be unique to AKT inhibitors. Since continual exposure to EGFR inhibitors in cell culture may elicit a heightened downstream activation of ERK phosphorylation, weekly dosing may similarly help to overcome these feedback loops (29).

There are a number of important questions raised by these results. i) Are the effects of weekly dosing with TKIs reproducible? The results with multiple EGFR inhibitors and the allosteric AKT inhibitor argues that it is reproducible. Furthermore, the recent preclinical data of Miller et al (30) with the PI3K inhibitor pictilisib argues that this may be a generalized property. Additionally, we recently found that gefitinib administered weekly was in fact more effective than daily dosing in both a carcinogen-induced model of lung adenocarcinomas as well as a human tumor xenograft employing an EGFR-mutant lung adenocarcinoma (31). ii) Although it is likely to be influenced by $\mathrm{PK}$ characteristics of individual agents, will the altered dosing regimen decrease toxicity? A reduction in toxicity may encourage the use of these agents in an adjuvant and even allow their use in a prevention setting. This question cannot be addressed in animal models since the rash or diarrhea observed in humans with these agents are not observed in rats or mice. However, effective weekly dosing with erlotinib for brain metastases of EGFR-mutant lung tumors is associated with a striking decrease in the incidence and severity of rash (25). Furthermore, a relatively large dose range study with erlotinib demonstrated the reduced toxicity of weekly dosing (26). This toxicity question can only be properly addressed clinically. However, the high prevalence of these symptoms would suggest that the toxicity aspects could be evaluated in relatively small clinical trials. iii) Is this greatly altered dosing applicable to multiple organ sites? Similarly, weekly dosing with gefitinib, erlotinib or lapatinib was as effective as daily dosing with the same agents in a rat urinary bladder cancer model (Lubet RA and Grubbs CJ, unpublished data). Finally, our studies found that weekly dosing of the PI3K inhibitor XL-147 or gefitinib also appeared more effective than daily dosing in a chemically induced lung cancer model (Wang Y, Lubet RA and You M, unpublished data).

\section{Acknowledgements}

The present study was supported by the NCI Contract No. HHSN261200433001C.

\section{Competing interests}

The authors declare that they have no competing interests.

\section{References}

1. Salamone DS, Brandt R, Ciardiello F and Normanno N Epidermal growth factor-related peptides and their receptors in human malignancies. Crit Rev Oncol Hematol 19: 183-232, 1995.

2. Arteaga CT: Overview of epidermal growth factor receptor biology and its role as therapeutic target in human neoplasia. Semin Oncol 29 (5 Suppl 14): S3-S9, 2002.
3. Moasser MM and Krop IE: The evolving landscape of HER2 targeting in breast cancer. JAMA Oncol 1: 1154-1161, 2015.

4. Hida L, Ogawa S, Park JC, Park JY, Shimazu J, Horio Y and Yoshida K: Gefitinib for the treatment of non small cell lung cancer. Expert Rev Anticancer Ther 9: 17-35, 2006.

5. Wong SF: Cetuximab: An epidermal factor receptor monoclonal antibody for treatment of colorectal cancer. Clin Ther 27: 684-694, 2005.

6. Tan WL and Ng QS: The continuing role of epidermal growth factor receptor tyrosine kinase inhibitors in advanced squamous cell carcinoma of the lung. Transl Lung Cancer Res 5: 106-109, 2016.

7. Polychronis A, Sinnett HD, Hadjiminas D, Singhal H, Masnsi JL, Shivapatham D, Shousha S, Jiang J, Peston D, Barrett N, et al: Preoperative gefitinib versus gefitinib and anastrozole in postmenopausal patients with oestrogen-receptor positive and epidermal-growth-factor-receptor-positive primary breast cancer: A double blind placebo controlled phase II randomized trial. Lancet Oncol 6: 383-391, 2005.

8. Baselga J, Albanell J, Ruiz A, Lluch A, Gascon P, Guillém V, González S, Sauleda S, Marimón I, Tabernero JM, et al: Phase II and tumor pharmacodynamic study of gefitinib in patients with advanced breast cancer. J Clin Oncol 23: 5323-5333, 2005.

9. Massarweh S, Thann YL, Huang J, Sexton K, Weiss H, Tsimelzon A, Beyer A, Rimawi M, Cai WY, Hilsenbeck S, et al: A phase II neoadjvant trial of anastrozole, fulvestrant, and gefitinib in patients with newly diagnosed estrogen receptor positive breast cancer. Breast Cancer Res Treat 129: 819-827, 2011.

10. Lubet RA, Szabo E, Iwata KK, Gill SC, Tucker C, Bode A, Steele VE, Juliana MM, Nicastro HL and Grubbs CJ: Effect of intermittent dosing regimens of erlotinib on methylnitrosoureainduced mammary carcinogenesis. Cancer Prev Res 6: 448-454, 2013.

11. Lubet RA, Szabo E, Christov K, Bode AM, Ericson ME, Steele VE, Juliana MM and Grubbs CJ: Effects of gefitinib (Iressa) on mammary cancers: Preventive studies with varied dosages, combinations with vorozole or targretin, and biomarker changes. Mol Cancer Ther 7: 972-979, 2008.

12. Li J, Cho YY, Langfald A, Carper A, Lubet RA, Grubbs CJ, Ericson ME and Bode AM: Lapatinib, a preventive/therapeutic agent against mammary cancer, suppresses RTK-mediated signaling through multiple signaling pathways. Cancer Prev Res 4: 1190-1197, 2011.

13. Ma CX, Sanchez C, Gao F, Crowder R, Naughton M, Pluard T, Creekmore A, Guo Z, Hoog J, Lockhart AC, et al: A Phase I Study of the AKT inhibitor MK-2206 in combination with hormonal therapy in postmenopausal women with estrogen receptor-positive metastatic breast cancer. Clin Cancer Res 22: 2650-2658, 2016.

14. Grubbs CJ, Peckham JC and McDonnough KD: Effect of ovarian hormones on the induction of 1-methyl-1nitrosourea induced mammary cancer. Carcinogenesis 4: 495-497, 1983.

15. Chan MM, Lu X, Merchant FM, Inglehart JD and Miron PL: Gene expression profiling of NMU-induced rat mammary tumors: Cross species comparison with human breast cancer. Carcinogenesis 26: 1343-1353, 2005.

16. Gottardis MM and Jordan VC: Antitumor actions of keoxifene and tamoxifen in the $N$-nitrosomethylurea-induced rat mammary carcinoma model. Cancer Res 47: 4020-4024, 1987.

17. Lubet RA, Steele VE, Casebolt TL, Eto I, Kelloff GJ and Grubbs CJ: Chemopreventive effects of the aromatase inhibitors vorozole (R-83842) and 4-hydroxyandrostenedione in the methylnitrosourea (MNU)-induced mammary tumor model in Sprague-Dawley rats. Carcinogenesis 15: 2775-2780, 1994.

18. Muller WJ, Sinn E, Pattengale PK, Wallace R and Leder P: Single-step induction of mammary adenocarcinoma in transgenic mice bearing the activiated c-neu oncogene. Cell 54: $105-115,1988$

19. Lu C, Speers C, Zhang Y, Xu X, Hill J, Steinbis E, Celestino J, Shen Q, Kim H, Hilsenbeck S, et al: Effect of epidermal growth factor receptor inhibitor on development of estrogen receptornegative mammary tumors. J Natl Cancer Inst 95: 1825-1833, 2003.

20. Zelazny E, Li B, Anagnostopoulos AM, Coleman A and Perkins AS: Cooperating oncogenic events in murine mammary tumorigenesis: Assessment of ErbB2, mutant p53, and mouse mammary tumor virus. Exp Mol Pathol 70: 183-193, 2001. 
21. Guttman-Yassky E, Mita A, De Jonge M, Matthews L, McCarthy S, Iwata KK, Verweij J, Rowinsky EK and Krueger JG: Characterization of the cutaneous pathology in (NSCLC) patients treated with (EGFR) tyrosine kinase inhibitor erlotinib. Eur J Cancer 46: 2010-2019, 2010.

22. Christov K, Shilkaitis A, Green A, Mehta RG, Grubbs C, Kelloff $\mathrm{G}$ and Lubet R: Cellular responses of mammary carcinomas to aromatase inhibitors: Effects of vorozole. Breast Cancer Res Treat 60: 117-128, 2000.

23. Lubet RA, Boring D, Steele VE, Ruppert JM, Juliana MM and Grubbs CJ: Lack of efficacy of the statins atorvastatin and lovastatin in rodent mammary carcinogenesis. Cancer Prev Res 2: 161-167, 2009.

24. Dowsett M, Smith IE, Ebbs SR, Dixon JM, Skene A, Griffith C, Boeddinghaus I, Salter J, Detre S, Hills M, et al: Short-term changes in Ki-67 during neoadjuvant treatment of primary breast cancer with anastrozole or tamoxifen alone or combined correlate with recurrence-free survival. Clin Cancer Res 11: 951S-958S, 2005.

25. Grommes C, Oxnard GR, Kris MG, Miller VA, Pao W, Holodny AI, Clarke JL and Lassman AB: 'Pulsatile' high-dose weekly erlotinib for CNS metastases from EGFR mutant nonsmall cell lung cancer. Neuro Oncol 13: 1364-1369, 2011.

26. Milton DT, Azzoli CG, Heelan RT, Venkatraman EM, Gomez JE, Kris MG, Krug LM, Pao W, Rizvi NA, Dunne M and Miller VA: A phase I/II study of weekly high-dose erlotinib in previously treated patients with nonsmall cell lung cancer. Cancer 107: 1034-1041, 2006.
27. Samadder NJ, Neklason DW, Boucher KM, Byrne KR, Kanth $P$, Samowitz W, Jones D, Tavtigian SV, Done MW, Berry T, et al: Effects of Sulindac and erlotinib vs placebo on duodenal neoplasia in familial adenomatous polyposis: A randomized clinical trial. JAMA 315: 1266-1275, 2016.

28. Chandarlapaty S, Sawai A, Scaltriti M, Rodrik-Outmezguine V, Grbovic-Huezo O, Serra V, Majumder PK, Baselga J and Rosen N: AKT inhibition relieves feedback suppression of receptor tyrosine kinase expression and activity. Cancer Cell 19: 58-71, 2011.

29. Ercan D, Xu C, Yanaita M, Monast CS, Pratilas CA, Montero J, Butaney M, Shimamura T, Sholl L, Ivanova EV, et al: Reactivation of ERK signaling causes resistance to EGFR kinase inhibitors. Cancer Discov 2: 934-947, 2012.

30. Yang W, Hosford SR, Dillon LM, Shee K, Liu SC, Bean JR, Salphati L, Pang J, Zhang X, Nannini MA, et al: Strategically timing inhibition of phosphatidylinositol 3-kinase to maximize therapeutic index in estrogen receptor alpha-positive PIK3CAmutant breast cancer. Clin Cancer Res 22: 2250-2260, 2016.

31. Zhang Q, Li R, Chen X, Lee SB, Pan J, Xiong D, Hu J, Miller MS, Szabo E, Lubet RA, et al: Effects of weekly or daily dosing regimen of Gefitinib in mouse models of lung cancer. Oncotarget 8: 72447-72456, 2017. 